\title{
UNIQUENESS IN THE SCHAUDER FIXED POINT THEOREM ${ }^{1}$
}

\author{
R. B. KELLOGG
}

\begin{abstract}
A condition is given which guarantees the uniqueness of the fixed point in the Brouwer and Schauder fixed point theorems. The result is applied to a nonlinear boundary value problem in physiology.
\end{abstract}

1. Let $X$ be a real Banach space with a bounded convex open subset $D$, and let $F: \bar{D} \rightarrow \bar{D}$ be a continuous function which is also assumed to be compact if $X$ is infinite dimensional. The Brouwer fixed point theorem (Schauder theorem if $X$ is infinite dimensional) gives a point $x \in \bar{D}$ such that $x=F(x)$. Under the assumption that $F$ is differentiable, we give a simple condition which guarantees that the fixed point $x$ is unique. The proof is an application of degree theory. We phrase the argument for the infinite dimensional case; the reader who is interested only in the finite dimensional case may omit the compactness hypothesis.

In the last section, the result is applied to a nonlinear boundary value problem arising in physiology.

2. Suppose that $F: \bar{D} \rightarrow \bar{D}$ is compact and continuously Fréchet differentiable in $D$. Then [4, Lemma 4.1] $F^{\prime}(x)$ is a compact linear operator on $X$ for each $x \in D$. Our uniqueness result is

THEOREM. Let $F: \bar{D} \rightarrow \bar{D}$ be a compact continuous map which is continuously Fréchet differentiable on $D$. Suppose that (a) for each $x \in D, 1$ is not an eigenvalue of $F^{\prime}(x)$, and (b) for each $x \in \partial D, x \neq F(x)$. Then $F$ has a unique fixed point.

In order to prove the Theorem we require a lemma. For the Lemma, recall that for any compact linear operator $A$ the spectrum, $\sigma(A)$, of $A$ consists of a countable number of points having 0 as the only possible limit point. Each nonzero $\lambda \in \sigma(A)$ is an eigenvalue of $A$. For such a $\lambda$, the null space of $(A-\lambda I)^{k}$ is, for all $k$ sufficiently large, independent of $k$. The dimension of this null space is called the algebraic multiplicity of the eigenvalue $\lambda$.

Lemma. Let $A$ be a compact linear operator on a real Banach space $X$. Suppose $1 \notin \sigma(A)$, and let $\beta(A)$ denote the sum of the algebraic multiplicities of all $\lambda \in \sigma(A)$ with $\lambda$ real and $\lambda>1$. Then there is an $\varepsilon>0$ such that if $B$ is a compact linear operator on $X$ and $\|A-B\|<\varepsilon$, then $(-1)^{\beta(A)}=(-1)^{\beta(B)}$.

Proof of Lemma. Let $B$ be a compact operator with $\|A-B\|<\varepsilon$, where $\varepsilon$ will be determined in the course of the proof. Letting $\varepsilon<1$, we see that

Received by the editors September 15, 1975 .

AMS (MOS) subject classifications (1970). Primary 47H10, 55C25, 34B15.

${ }^{1}$ This work was supported by the National Institutes of Health under contract \#HI52900.

Cupyright $\odot 1977$, American Mathematical Suciety 
$\|B\|<M=\|A\|+1$. Let $Q$ be an open rectangle in the $\lambda$ plane with two sides on $\operatorname{Re} \lambda=1$ and $\operatorname{Re} \lambda=M$, and the other two sides on $\operatorname{Im} \lambda= \pm a$. Let $\Gamma$ denote the boundary of $Q$. We pick $a>0$ so small that $\sigma(A) \cap \bar{Q}$ consists only of real eigenvalues. Then $\Gamma \cap \sigma(A)=\varnothing$. For $\varepsilon$ sufficiently small, we have $\Gamma \cap \sigma(B)=\varnothing\left[2\right.$, p. 213]. Let $R_{A}(\lambda)=(\lambda I-A)^{-1}$ be the resolvant operator of $A$, and set

$$
P_{A}=\int_{\Gamma}(\lambda I-A)^{-1} d \lambda
$$

Then $P_{A}$ is a projection whose range is the union of the eigenspaces of $A$ corresponding to the eigenvalues $\lambda \in Q \cap \sigma(A)$ [2, p. 178]. Thus, setting $d\left(P_{A}\right)=$ the dimension of the range of $P_{A}$, we find that $d\left(P_{A}\right)$ is the sum of the algebraic multiplicities of the eigenvalues $\lambda \in \sigma(A) \cap Q$. Defining $P_{B}$ in a similar way, we have a similar result for $d\left(P_{B}\right)$. For $\varepsilon$ sufficiently small, we have $\left\|P_{A}-P_{B}\right\|<1$, so $d\left(P_{A}\right)=d\left(P_{B}\right)[2$, p. 33]. Since $\sigma(A) \cap Q$ consists only of real eigenvalues, and since $\lambda \in \sigma(A)$ satisfies $|\lambda|<M$, we have $d\left(P_{A}\right)=\beta(A)$. If $\lambda \in \sigma(B)$ is complex, then $\bar{\lambda} \in \sigma(B)$, and $\lambda$ and $\bar{\lambda}$ have the same algebraic multiplicity. Hence $d\left(P_{B}\right)=\beta(B)+$ even number. Combining these facts, we find that $\beta(A)$ and $\beta(B)$ have the same parity, which proves the lemma.

Proof of Theorem. We first show that $F$ has a finite number of fixed points. For supposing otherwise, let $x^{k}=F\left(x^{k}\right), k=1,2, \ldots$, be a sequence of fixed points. Since $F$ is compact we may, after picking a subsequence, suppose that $x^{k} \rightarrow x \in \bar{D}, F(x)=x$. Hence $x \in D$. By the spectral condition, $I-F^{\prime}(x)$ has a bounded inverse, so from the inverse function theorem [5, Theorem 1.20], $I-F(x)$ is (1-1) in a neighborhood of $x$, contradicting $(I-F)\left(x^{j}\right)=0$. Let $x^{1}, \ldots, x^{N}$ denote the fixed points of $F$. Let $U_{j}$ be a neighborhood of $x^{j}$ such that the closed sets $\bar{U}_{j}$ are pairwise disjoint and $\bar{U}_{j} \subset D$. Let $K=\bar{D} \backslash\left\{\cup_{1}^{N} U_{j}\right\}$, so $K$ is a closed subset of $\bar{D}$ which does not contain a fixed point of $F$. Then the quantities

$$
\operatorname{deg}(0, I-F, D) \text { and } \operatorname{deg}\left(0, I-F, U_{j}\right)
$$

are well defined, and from the excision and additive properties of the degree [5, Proposition 3.37], we have

$$
\operatorname{deg}(0, I-F, D)=\operatorname{deg}(0, I-F, D \backslash K)=\sum_{j} \operatorname{deg}\left(0, I-F, U_{j}\right) .
$$

Without loss of generality we may suppose $0 \in D$. Then $H(x, t)=x-$ $t F(x), 0 \leqslant t \leqslant 1$, defines a homotopy of $F$ with the identity function $I$. Since $0 \in D, H(x, t) \neq 0$ for $x \in \partial D$, for otherwise $x=t F(x)+(1-t) 0 \in D$, which is a contradiction. Hence $\operatorname{deg}(0, I-t F, D)$ is defined, so using this homotopy,

$$
\operatorname{deg}(0, I-F, D)=\operatorname{deg}(0, I, D)=1 .
$$

From [4, Theorem 4.7],

$$
\operatorname{deg}\left(I-F, 0, U_{j}\right)=(-1)^{\beta\left(x_{j}\right)}
$$

where, in the notation of the Lemma, $\beta(x)=\beta\left(F^{\prime}(x)\right)$. Since $F^{\prime}(x)$ is 
continuous, we see from the Lemma that the parity of $\beta(x)$ is constant for $x \in D$. Hence $1= \pm N$, so $N=1$ and the fixed point is unique.

REMARKS. (1) The same argument gives a uniqueness condition for the fixed point theorems of Altman and Rothe [5, Chapter 3]. (2) We thank Dr. John Osborn for help in proving the Lemma.

3. To illustrate our result, we consider the nonlinear boundary value problem

$$
\begin{aligned}
& \left\{\begin{array}{l}
-D C^{\prime \prime}+(v C)^{\prime}=f(x), \quad 0<x<1, \\
C^{\prime}(0)=0, \quad C(1)=a>0,
\end{array}\right. \\
& v^{\prime}=-J(x, C), \quad v(0)=0 .
\end{aligned}
$$

This system of equations was used by Diamond and Bossert [1] to model salt and water transport in a closed-ended tube, such as a sweat gland. The diffusion coefficient $D>0$, the function $J(x, C)$ represents an osmotic transport of water out of the tube, and the function $f(x)$ represents a source of salt into the tube. We assume that these functions are sufficiently differentiable, and that

$$
f(x) \geqslant 0, \quad J_{C}(x, C)<0,
$$

where the subscript $C$ denotes the partial derivative.

We write (1), (2) as a fixed point problem as follows. Let $C_{1}(x)$ be a continuous function, and with $C=C_{1}$, let $v(x)$ be the solution of (2). Then with $v(x)$ given, there is a unique solution $C=C_{2}$ of (1). To see this, we integrate (1) to obtain

$$
-D C_{2}^{\prime}(x)+v(x) C_{2}(x)=f_{1}(x)=\int_{0}^{x} f(t) d t .
$$

Letting $v_{1}$ denote an indefinite integral of $v$, we may then solve this equation to get

$$
\begin{aligned}
C_{2}(x)= & a \exp \left\{-D^{-1}\left[v_{1}(1)-v_{1}(x)\right]\right\} \\
& +D^{-1} \int_{x}^{1} f_{1}(t) \exp \left\{-D^{-1}\left[v_{1}(t)-v_{1}(x)\right]\right\} d t
\end{aligned}
$$

We have thus defined a map $F\left(C_{1}\right)=C_{2}$ on the Banach space $X$ of continuous functions on $[0,1]$.

The problem of solving the system (1), (2) is equivalent to the problem of finding a fixed point of $F$. From (4) and (3) we see that $C_{2}(x)>0$. If $C_{1}(x) \geqslant 0$, we have from $(3), v(x) \geqslant w(x)$, where $w(x)=-\int_{0}^{x} J(t, 0) d t$. Hence for $t \geqslant x, v_{1}(t)-v_{1}(x) \geqslant b(t-x)$, where $b$ is independent of $C_{1}(x)$. Using this in (4), we find that there is a constant $K$ such that, for any $C_{1}(x) \geqslant 0, C_{2}(x)<K$. If we let $D \subset X$ denote the convex set defined by the inequalities $0<C(x)<K$, we have proved that $F(\bar{D}) \subset D$. It is easy to see, using (4), that $F$ is continuous, compact, and in fact continuously Fréchet differentiable. Thus from the Schauder fixed point theorem, there is a fixed point $C=F(C)$, and hence a solution $v(x), C(x)$, of (1), (2). To show that there is a unique fixed point, we must calculate the derivative of $F$. Setting $C_{2}=F\left(C_{1}\right), \tilde{C}_{2}=F^{\prime}\left(C_{1}\right) \tilde{C}_{1}$, it may be verified that $\tilde{C}_{1}, \tilde{C}_{2}$ satisfy the linear 
problem

$$
\begin{array}{rlrl}
-D \tilde{C}_{2}^{\prime \prime}+\left(\tilde{v} C_{2}\right)^{\prime}+\left(v \tilde{C}_{2}\right)^{\prime} & =0, & \tilde{C}_{2}^{\prime}(0)=\tilde{C}_{2}(1)=0 \\
\tilde{v}^{\prime}+J_{C}\left(x, C_{1}\right) \tilde{C}_{1}=0, & \tilde{v}(0)=0 .
\end{array}
$$

To apply our Theorem, we suppose $C_{1} \in D$. We must verify that $1 \notin$ $\sigma\left(F^{\prime}\left(C_{1}\right)\right)$; that is, that 1 is not an eigenvalue of $F^{\prime}\left(C_{1}\right)$. Supposing the contrary, let $\tilde{C}$ be an eigenfunction of $F^{\prime}\left(C_{1}\right)$ with eigenvalue 1 . Then $\tilde{C}_{2}=\tilde{C}$, and hence there is a nontrivial solution $\tilde{v}(x), \tilde{C}(x)$, of the problem

$$
\begin{gathered}
-D \tilde{C}^{\prime \prime}+\left(\tilde{v} C_{2}\right)^{\prime}+(v \tilde{C})^{\prime}=g_{1}(x), \\
\tilde{v}^{\prime}+J_{C}\left(x, C_{1}\right) \tilde{C}=g_{2}(x), \\
\tilde{C}^{\prime}(0)=\tilde{C}(1)=\tilde{v}(0)=0
\end{gathered}
$$

with $g_{1}(x) \equiv 0, g_{2}(x) \equiv 0$. It may be verified that $(5)-(7)$ defines a closed, densely defined operator on $(\tilde{v}, \tilde{C}) \in L_{2}(0,1) \times L_{2}(0,1)$, that the resolvent operator is compact, and that the adjoint operator is given by the solution of the problem

$$
\begin{gathered}
-\varphi^{\prime}-C_{2} \psi^{\prime}=h_{1}(x), \\
-D \psi^{\prime \prime}-v \psi^{\prime}+J_{C} \varphi=h_{2}(x), \\
\psi^{\prime}(0)=\psi(1)=\varphi(1)=0 .
\end{gathered}
$$

Using the Fredholm alternative and the compactness of the resolvent operator associated with the problem (5)-(7), we conclude that there are functions $\varphi(x), \psi(x)$, not identically zero, which satisfy (8)-(11) with $h_{1}(x) \equiv 0, h_{2}(x)$ $\equiv 0$. We must have $\psi^{\prime}(1) \neq 0$, since otherwise, by the uniqueness of the solution of the terminal value problem associated with (8), (9), we would have $\varphi \equiv 0, \psi \equiv 0$. Let $\bar{x}<1$ be the largest zero of $\psi^{\prime}$, and suppose that $\psi^{\prime}(x)>0$ in $(\bar{x}, 1)$. Since $C_{2}=F\left(C_{1}\right) \in D$, we have $C_{2}(x)>0$ in $[0,1]$. Hence from (8), $\bar{x}$ is the largest zero of $\varphi^{\prime}$, and $\varphi^{\prime}(x)<0$ in $(\bar{x}, 1)$. Hence $\psi^{\prime \prime}(\bar{x}) \geqslant 0$, $\varphi(\bar{x})>0$. Using (3), we see that the left side of (9) is $<0$ at $x=\bar{x}$. This is a contradiction and proves that 1 is not an eigenvalue of $F^{\prime}\left(C_{1}\right)$. Hence by our Theorem, there is a unique fixed point of $F$, and a unique solution $v(x), C(x)$ of (1), (2) with $C(x) \geqslant 0$.

REMARK. In [3] there is given a more detailed study of osmotic flow in a tube.

\section{REFERENCES}

1. J. M. Diamond and W. H. Bossert, Standing-gradient osmotic flow, J. General Physiology 5 (1967), 2061-2083.

2. Tosio Kato, Perturbation theory for linear operators, Springer-Verlag, New York, 1966. MR 34 \#324.

3. R. B. Kellogg, Osmotic flow in a tube with stagnant points, Technical Note BN-818, IFDAM, Univ. of Maryland, College Park, Md., July, 1975.

4. M. A. Krasnosel'skii, Topological methods in the theory of nonlinear integral equations, GITTL, Moscow, 1956; English transl., Macmillan, New York, 1964. MR 20 \#3464; 28 \#2414.

5. J. T. Schwartz, Non-linear functional analysis, Gordon and Breach, New York, 1969.

Institute for Fluid Dynamics and Applied Mathematics, University of Maryland, College Park, Maryland 20742 